\title{
Patient-centered care retreats as a method for enhancing and sustaining compassion in action in healthcare settings
}

Sara Guastello ${ }^{1 *}$ and Susan B Frampton ${ }^{2}$

\begin{abstract}
Background: Compassion, and its close counterparts empathy and kindness, are increasingly being recognized as essential dimensions of a quality health care experience, with further evidence demonstrating that they can be developed behaviors. This has spurred the creation of a number of curricula and tools for teaching empathy and compassionate communication, however many of these curricula approach this work from a task orientation, neglecting to address the important dimension of organizational culture.
\end{abstract}

Case description: The Planetree patient-centered care retreat, first delivered in 1985, combines skill-development with inspiration and team-building to support staff in connecting to their deeper motivations for being a caregiver. It offers tools and support for nurturing compassion among all caregivers and leaders, and for embedding compassionate human interactions into organizational processes and practices. Though there is ample room for customization of the retreat curriculum to meet specific organizational needs, common elements of the Planetree patient-centered care retreat include:

- In-person delivery to promote team-building and human connections

- Experiential in nature

- Facilitated by peers from within the organization

- Attended by a co-mingled staff group from a variety of departments/sectors of the organization.

Discussion and Evaluation: The experience of health care organizations that have committed to sending a critical mass of employees through the retreat process suggests that the dual focus on inspiration and skills-building drives improved outcomes related to both the patient and employee experience.

Conclusions: These outcomes result from a curriculum and program structure specially designed to cultivate change agents within an organization. The retreat is a collective experience, binding employees who perform a variety of functions from different sectors of the organization around a common purpose and vision for how care is to be delivered. Optimally, as a critical mass of employees participates in this shared experience the momentum to bring about the change envisioned during the retreats builds until providing compassionate, patient-centered care is no longer the exception, but the expectation within the organization.

Keywords: Compassion, Patient-centered care, Retreat, Planetree, Patient and family engagement

\footnotetext{
* Correspondence: sguastello@planetree.org

'Director of Knowledge Management, Planetree, 130 Division Street, Derby

CT 06418, UK

Full list of author information is available at the end of the article
} 


\section{Background}

Compassion, and its close counterparts empathy and kindness, are increasingly being recognized as essential dimensions of a quality health care experience that drives improved health outcomes [1-5], enhanced patient satisfaction and loyalty [6], and even reduced rates of litigation [7]. These associations are not surprising. Often the interactions that occur between patients (and their loved ones) with their health care providers happen at among the most sensitive and vulnerable times during a person's life. When these interactions transpire with an underpinning of compassion and caring attitudes, it establishes a basis for a trusting relationship that encourages patient and family engagement (PFE), now seen as a fundamental determinant of quality outcomes [8-12], reduced utilization [13,14] and lower costs [15].

This connection between the quality and tenor of human interactions and PFE was reinforced in a 2013 survey on kindness in health care. More than half of the respondents reported that when they experience a lack of kindness in a health care setting, they withhold information from their health care providers [16]. Conversely, evidence demonstrates that when care is delivered with compassion and empathy, patients talk more openly about their symptoms and concerns, which enables their clinicians to better assess their condition and make more accurate diagnoses [17].

\section{Compassion as a core competency for all caregivers}

Despite this fairly basic and intuitive premise-that compassion is an important dimension of any health care experience-practical, field-tested strategies for facilitating compassionate human interactions in health care settings are limited. Perhaps these limitations have been fueled by a mistaken presumption that compassion, empathy and kindness are not learned behaviors. Emerging research, however, suggests that this is not the case, and indeed "certain awareness-building and reflection activities seem to be able to up-regulate empathic behavior" [18]. As the evidence base for compassion as a mutable trait with the potential to improve outcomes and enhance quality grows, approaches for embedding empathic communication techniques and compassionate behaviors into routine health care interactions are becoming more prevalent [19-23].

A precursor to this relatively recent modern-day heightened consciousness to the importance of compassion in health care delivery is the Planetree methodology for patient-centered care (PCC) that prioritizes openness, partnership, mutual respect and compassion, and involvement of family as key factors for engaging patients in managing their own care and modifying their health behaviors [24].
Planetree is a not-for-profit advocacy, education and membership organization working to advance patientcentered care delivery across the health care continuum for more than thirty-five years [25]. The Planetree approach is informed by the experiences of those who interact firsthand with the health care system-as patients, family members and professional caregivers. Drawing on this knowledge base and Planetree focus group data [26] that consistently demonstrates the importance to patients in their health care encounters of feeling valued and respected, having a sense of control and being provided with opportunities to participate in their care, one of the defining components of the Planetree methodology is Compassionate Human Interactions. This component emphasizes that the foundation of a healing system is the relationships and interactions that occur among patients, families and health care givers [27]. A central tenet of the Planetree philosophy is that quality human interactions are based on each caregiver's ability to be present to self and to others, and to show compassion.

The methodology stresses organizational culture change that goes beyond structures, programs and tactics to engage the hearts and minds of an entire team around a common vision for how we partner with patients and engage their families. This organizational culture change work is grounded in an understanding that discrete PCC implementation tactics are of little use if they are rolled out in an environment that has not addressed the quality of basic human interactions.

\section{Case presentation: patient-centered care retreats}

A hallmark element of the Planetree methodology is the patient-centered care retreat, named as such to differentiate it from the traditional types of skill-building workshops, lectures, and didactic training offered abundantly in most health care settings. The retreat curriculum is built around experiential activities, discussion prompts, "who are you really?" group sharing, and personal reflection exercises, which all build on each other to advance the overriding goal of ensuring that compassionate human interactions do not become an afterthought in the midst of task-driven shifts, challenging organizational times, high census days and difficult patient care situations. Patient-centered care retreats - whether offered for new employees as part of the onboarding process or as "refreshers" for long-time staff-offer the opportunity for all health care professionals, inclusive of administrative and clinical leadership, front-line staff, support services, contract workers, even volunteers, to come together for the unique opportunity to reflect on why what they do matters; and even more so, why how they do what they do matters even more.

The first retreat was held in January 1985. Over the intervening years, health care organizations around the 
world implementing the Planetree model have customized the retreat to make it their own, but nonetheless, the guiding principles of the retreat have not changed:

- They occur in-person (versus virtually) to promote team-building and human connections.

- Experiential exercises are heavily featured (not just lecture and reading).

- They are facilitated by peers from within the organization, trained in facilitation skills via a train-the-trainer approach.

- They are attended by a co-mingled staff group from a variety of departments/sectors/tiers of the organization.

This interdisciplinary composition is an important differentiator of the retreats from other types of empathy training or compassion curricula that tend to target a specific audience, oftentimes clinical staff. By inviting frontline staff to participate alongside senior executives, physicians and staff from support or ancillary services, the retreats underscore that in an organizational culture of PCC, everyone is a caregiver, with the expectation and accountability for embodying compassion in action in their work-whatever that work may be. After all, from the patient's perspective, the title or job description of someone extending a kind gesture, communicating with compassion or empathy, or exhibiting a caring attitude is of considerable less importance than that the caregiver has connected with him or her on a human level. Furthermore, this co-mingling of staff at the retreats establishes a basis for teamwork that extends beyond any formal interdisciplinary committees or collaborations. As evidenced by these comments from staff who participated in a retreat, the non-traditional composition of the groups does not go unnoticed:

"I think it's great that it's everyone from every area. It makes a difference when you interact with someone."

\section{"The Planetree retreat was amazing, and you get to know everyone in your group. It was beautiful. It made Stamford Hospital feel like a family." \\ "It makes it cohesive. I still see people that I went through with, and we still have the same connection from our retreat. There are all levels; I came through with the CIO and other directors. It's just neat."}

\section{Experiential exercises}

An emphasis on experiential exercises is a defining feature of the retreats. These activities are designed to connect participants to the importance of the principles of patient-centered care in a more demonstrable and personal way, which better enables them to retain the content. A purely didactic approach does not have the same impact.
Examples of experiential components incorporated into the retreats include a "Walk in the Patient's Shoes" exercise in which participants are paired up, with one blindfolded and an assigned partner escorting them in silence in a "trust walk." The escort uses non-verbal cues and the power of touch to build rapport and trust with their blindfolded partner. Trained facilitators debriefing the exercise with participants relate the experience of the blindfolded partner to how it feels from the patient perspective to experience the unknown (e.g. what happens next? Who is my caregiver? Am I in good hands? Is my environment safe?). The escort is encouraged to examine how it makes them feel to assume total responsibility for a person's wellbeing. An extension of this exercise has those guiding the blindfolded participants switch off partners to demonstrate the impact of having one's care handed off to another caregiver and the importance of making those handoffs as seamless as possible.

\section{Adaptations}

Planetree estimates that a version of its retreat curriculum has been delivered in hundreds of health care organizations (including hospitals, long-term care communities, behavioral health hospitals, integrated health care systems, ambulatory settings and physician practices) in diverse cultures around the world. Given the diversity in the settings implementing the curriculum, it is not surprising that a number of adaptations have emerged over the years.

The hallmark retreat is an off-site, two-day, overnight endeavor, which allows the retreat facilitators to parallel the experience of being away from home, separated from loved ones, and in close quarters to a stranger (their assigned roommate) to the patient experience. In these economically challenging times, many organizations simply find that they cannot bear the costs of sending small groups of staff off-site for two days, along with the associated costs of providing coverage for the retreat participants. Alternatively, the retreat content can be abbreviated to accommodate the desired duration, be it a full-day, half-day, or a series of shorter modules offered over a period of time. Other sites have expanded the duration of the retreats, incorporating a community service project as a culminating team-building activity.

Hosting the retreat off-site enables staff to more fully disconnect from their daily work tasks and be present for the retreat activities. Some organizations have been able to achieve this same aim at lower cost by designating a space on campus for the retreat and enhancing it with fun or inspirational décor that sends the message that this is not just another day at the office.

For long-term care organizations endeavoring to transform their cultures to one of resident-directed, relationship-centered care, modifying the content and exercises from the traditional staff retreat for the resident 
population has been a powerful way to create a stronger sense of community among those who live and work there. Activities are designed to sensitize residents to the experiences of those they live with, to promote their active engagement as problem-solvers and to encourage each resident to get back in touch with who they are outside of preconceived notions related to age, illness or disability [28].

\section{Inspiration alone is not enough}

Inspiration alone is not enough to power the kind of organizational culture change necessary to realize the benefits of patient-centered care. Poignant stories, motivational team building activities, and exercises that place staff in the shoes of their patients may help to fuel a collective sense of purpose that pervades the organization, but that sense of purpose will disintegrate if retreat participants are not also provided with practical tools and dayto-day implementation strategies for converting their reignited passion for delivering compassionate, patientcentered care into an operational reality they can deliver on. Accordingly, the most impactful retreats balance inspiration with education and competency-building around communication and technical skills.

\section{Discussion and evaluation}

The impact of the retreat is evident in the experiences of numerous Planetree affiliates. Though at this time no large-scale evaluation to examine the relationship between implementation of retreats and quality outcomes has been undertaken, the site-specific data presented below suggest that retreats are a powerful lever for measurable change within an organization. Stamford Hospital, a 305-bed tertiary care center in Stamford, Connecticut, adopted an aggressive retreat participation goal. Within eighteen months of introducing the Planetree model, $90 \%$ of employees had participated in the full-day, off-site retreat experience. At the end of the 18-month period, the hospital had realized significant gains in employee satisfaction (from the 33rd to 60th percentile), emergency department patient satisfaction (from the 44th to the 89th percentile) and inpatient satisfaction (from the 18th to the 75th percentile) [29].

Over the course of two years, more than $94 \%$ of the 950 full-time employees at Carolinas Medical CenterMercy, a 180-bed hospital, participated in the hospital's retreat. Within two years, the hospital's performance on the "likelihood to recommend" question on its patient experience survey increased from $61 \%$ to $80.8 \%$. At the same time, $90 \%$ of employees participated in the employee engagement survey and the workforce commitment indicator score rose to the 89th percentile. In order to sustain those gains, the hospital has continued to offer "refresher" retreats for graduates of the initial retreat to maintain momentum and enthusiasm, and to keep compassionate human interactions top of mind for all employees [30].

The value of these accumulative retreats is echoed in the experience of New York Presbyterian Hospital/ Westchester Division, a freestanding behavioral health hospital in White Plains, New York. Since 2004, the hospital has offered a retreat experience approximately every two years for its employees. Building on earlier retreat content, each subsequent iteration of the retreat reinforces the concepts of patient-centered care, and strengthens the organizational culture of teamwork and open communication. Concurrently (from 2005 to 2013), patient satisfaction has increased from $78 \%$ to $85 \%$ and employee satisfaction has increased from $78 \%$ to $91 \%$ [31].

\section{A linchpin for achieving patient-centered excellence}

Each of the three hospitals highlighted in the section above has been recognized as an exemplary patientcentered hospital through Planetree Patient-Centered Hospital Designation Program. A comprehensive program for advancing and assessing patient-centered excellence, the Patient-Centered Hospital Designation Program serves as an implementation framework for the complex work of organizational culture change [32,33]. Drawing on extensive focus group research, along with the knowledge derived from partnering with organizations in the field to develop strategies for meeting patient and staff needs, Planetree launched the program in 2007 to support organizations in creating and maintaining a patient-centered and compassionate culture. The program addresses core components of culture change and PCC with explicit organizational performance criteria, effectively converting patient-centered care from an aspiration into a goal that can be set, measured and attained.

Among the more than 50 criteria an organization must fulfill to achieve Designation is criterion II.A.:

\section{II.A: All staff members of the primary organization being recognized, including off-shift, part-time, and support staff are given an opportunity to participate in a patient-/resident-centered staff retreat experience or a comparable experiential patient-centered care immersion program, with a minimum concurrent completion rate of $85 \%$. In addition, per diem staff, employed medical staff and other providers (physician assistants, nurse practi- tioners and clinical nurse specialists) and volunteers are encouraged to participate in a retreat experience [34].}

The inclusion of this criterion from the very onset of the program derived from the insights of numerous leaders from hospitals with established reputations as being patient-centered (evidenced by long-term commitment to establishing PFE practices resulting in high patient and employee satisfaction). These leaders consistently 
credited having a critical mass of their employee base participate in a retreat as a defining milestone of their patientcentered care implementation journey, and one that differentiated for staff patient-centered care as a cultural shift (how we do things) from the more familiar types of programmatic intervention (what we do).

Providing evidence of retreat participation is not sufficient for an organization to fulfill the criterion. An on-site assessment includes focus groups with recently discharged and current patients, family members, members of the medical staff, and employees to validate that the spirit and intent of each criteria are reflected back in the lived experiences of these key stakeholder groups. Confirmation of attendance by employees in focus groups does not demonstrate fulfillment either. Focus group questions probe how the retreat experience connected staff to what it means to deliver compassionate, patient-centered care, and how it has shaped the organization's culture and their interactions with patients and families, as well as with each other. Comments from staff in focus groups held at sites that ultimately were awarded the designation include:

\section{"As a new employee that first week, I went to the retreat. This is more than anything I had experienced...it's not the building that makes the institution it's the people. Always willing to lend a hand."}

"I went on the retreat a few weeks after coming here. It showed us how different the hospital is from others. I worked somewhere else and can't remember talking about the family or ideas or feedback."

\section{"It's that whole experience from even before entering the hospital to post-discharge, and seeing it through the patient or family eyes. I was asked to be a fake patient in the chest pain center. I was in the ER and did a mock drill. Until then, I was the nurse pushing those patients fast through the halls, but until you are lying there, you don't realize what it's like and how fast they push you! It puts you in their place and you see what they see. It gives a whole new perspective."}

\section{Future developments}

Currently, one of the great challenges facing health care professionals today is a nearly universal demand to provide more complex care in shorter lengths of time with fewer resources-all without compromising quality and safety. With this heightened focus on efficiency, the ability of an organization to release staff for a full day (and potentially more) to participate in a patient-centered care retreat is a significant barrier. Given this, further examination is needed to consider how the baseline retreat curriculum can be modified in such a way to allow for meaningful engagement with the content in shorter periods of time, and potentially in a virtual environment-without conceding the very elements of the retreat that make it so powerful and impactful.

In addition, opportunities exist for using outcomes data to strengthen the case for patient-centered care retreats. Any organization implementing retreats is encouraged to track participation rates, as well as to have all participants complete a program evaluation to solicit their input and reactions to the curriculum. In addition, it is recommended that the participation figures are correlated with employee engagement and patient satisfaction scores over time to explore the relationship between percentage of employees participating in the retreat and improvements in both the patient and employee experience.

\section{Conclusion}

Empirical research is now validating the intuitive conviction that compassion is an essential component of quality care. Good intentions alone, though, will not manifest an organizational culture of compassion. A focused, intentional effort must be undertaken to train and support staff to undertake their work-whatever that work may be-with attitudes of empathy, caring and kindness, and to forge meaningful partnerships with patients and families. A method for doing just that, the Planetree patient-centered care retreat combines inspiration with education and training to support staff in connecting to their deeper motivations for being a caregiver and offering up the tools and support for nurturing and eliciting compassion among all caregivers and leaders, and embedding compassionate human interactions into organizational processes and practices.

By so doing, the retreats cultivate change agents within an organization. The retreat is a collective experience, binding employees who perform a variety of functions from different sectors and tiers of the organization around a common purpose. As a critical mass of employees participates, the momentum to bring about the change envisioned during the retreats builds until providing compassionate, patient-centered care is no longer the exception, but the expectation within the organization.

\section{Abbreviations}

PFE: Patient and family engagement; PCC: Patient-centered care.

\section{Competing interests}

The authors declare they have no competing interests.

\section{Authors' contributions}

SG conceived of the case study, consolidated experiences and findings across sites implemented the retreat and drafted the manuscript. SF conceived of the case study and helped to draft the manuscript. Both authors read and approved the final manuscript.

\section{Authors' information}

SG is the director of knowledge management with Planetree, where she has developed a range of educational and implementation resources to guide organizations in their endeavors to deliver patient-centered care. She also 
oversees operations of the Patient-Centered Hospital Designation Program ${ }^{\circledast}$. SF is the president of Planetree, a not-for-profit advocacy, consulting and membership organization that promotes patient-centered care.

\section{Author details}

'Director of Knowledge Management, Planetree, 130 Division Street, Derby CT 06418, UK. ²President, Planetree, 130 Division Street, Derby CT 06418, UK.

Received: 1 April 2014 Accepted: 27 June 2014

Published online: 11 November 2014

\section{References}

1. Alexander JA, Hearld LR, Mittler JN, Harvey J: Patient Physician Role Relationships and Patient Patient Activation Among Individuals with Chronic Illness. Health Serv Res 2012, 47(3 Pt 1):1201-1223.

2. Beach MC, Keruly J, Moore RD: Is the Quality of the Patient-Provider Relationship Associated with Better Adherence and Health Outcomes for Patients with HIV? J Gen Intern Med 2006, 21(6):661-665.

3. Haslam N: Humanizing medical practice: the role of empathy. Med J Aust 2007, 187(7):381-382.

4. Hojat M, Louis DZ, Markham FW, Wender R, Rabinowitz C, Gonnella JS: Physicians' empathy and clinical outcomes for diabetic patients. Acad Med 2011, 86(3):359-364

5. Rakel D, Barrett B, Zhang Z, Hoeft T, Chewning B, Marchand L, Scheder J: Perception of empathy in the therapeutic encounter: effects on the common cold. Patient Educ Couns 2011, 85(3):390-397.

6. Safran DG, Montgomery JE, Chang H, Murphy J, Rogers WH: Switching doctors: predictors of voluntary disenrollment from a primary physician's practice. J Fam Pract 2001, 50(2):130-136.

7. Virshup BB, Oppenberg AA, Coleman MM: Strategic risk management: reducing malpractice claims through more effective patient-doctor communication. Am J Med Qual 1999, 14(4):153-159.

8. Greene J, Hibbard $\mathrm{JH}$ : Why does patient activation matter? An examination of the relationships between patient activation and health-related outcomes. J Gen Intern Med 2012, 27(5):520-526.

9. Harvey $L$, Fowles JB, Xi M, Terry P: When activation changes, what else changes? the relationship between change in patient activation measure (PAM) and employees' health status and health behaviors. Patient Educ Couns 2012, 88(2):338-343.

10. Hibbard JH, Mahoney ER, Stock R, Tusler M: Do increases in patient activation result in improved self-management behaviors? Health Serv Res 2007, 42(4):1443-1463.

11. Remmers C, Hibbard J, Mosen DM, Wagenfield M, Hove RE, Jones C: Is Patient Activation Associated with Future Health Outcomes and Healthcare Utilization Among Patients with Diabetes? J Ambul Care Manage 2009, 32(4):1-8.

12. Skolasky RL, Mackenzie EJ, Wegener ST, Riley LH: Patient activation and functional recovery in persons undergoing spine surgery. Orthopedics 2011, 34(11):888

13. Stacey D, Bennett CL, Barry MJ, Col NF, Eden KB, Holmes-Rovner M, Llewellyn-Thomas $H$, Lyddiatt A, Légaré F, Thomson R: Decision aids for people facing health treatment or screening decisions. Cochrane Database Syst Rev 2011, 10:CD001431. Review. Update in: Cochrane Database Syst Rev. 2014.

14. Wennberg DE, Marr A, Lang L, O'Malley S, Bennett G: A randomized trial of a telephone care-management strategy. N Engl J Med 2010, 363(13):1245-1255.

15. Hibbard JH, Greene J, Overton V: Patients with lower activation associated with higher costs; Delivery systems should know their patients' 'scores. Health Aff 2013, 32(2):216-222.

16. Greensweig G: Why Practicing medicing with kindness matters. In Becker's Hospital Review; 2014. http://www.beckershospitalreview.com/leadershipmanagement/why-practicing-medicine-with-kindness-matters.html.

17. Epstein RM, Franks P, Shields CG, Meldrum SC, Miller KN, Campbell TI, Fiscella K: Patient-centred communication and diagnostic testing. Ann Fam Med 2005, 3:415-421.

18. Riess H, Kelley JM, Bailey RW, Dunn EJ, Phillips M: Empathy training for resident physicians: a randomized controlled trial of a neuroscienceinformed curriculum. J Gen Intern Med 2012, 27(10):1280-1286.

19. Dewar B, Cook F: Developing compassion through a relationship centred appreciative leadership programme. Nurse Educ Today 2014, 34(9):1258-1264.
20. Frankel RM, Stein T: Getting the most out of the clinical encounter: the four habits model. J Med Pract Manage 2001, 16(4):184-191.

21. Jazaieri H, Jinpa G, McGonigal K, Rosenberg E, Finkelstein J, Simon-Thomas E, Cullen M, Doty J, Gross J, Goldin P: J Happiness Stud 2013, 14(4):1113-1126.

22. Millenson ML: Building a better care relationship with effective doctorpatient communication. Group Pract J 2012, 61:12-16.

23. Scott KW, Phil M, Jha AK: Putting Quality of the Global Health Agenda. N Engl J Med 2014, 371(1).

24. Planetree. www. planetree.org.

25. Frampton SB, Charmel PA, Guastello S: The Putting Patients First Field Guide Global Lessons in Designing and Implementing Patient-Centered Care. San Francisco: Jossey-Bass; 2013.

26. Planetree and Picker Institute: Patient-Centered Care Improvement Guide. Derby: CT and Camden, ME; 2008

27. Frampton SB, Charmel PA: Putting patients first: designing and practicing patient-centered care. 2nd edition. San Francisco: Jossey-Bass; 2008.

28. Planetree and Picker Institute: Long-Term Care Improvement Guide. Derby: CT and Camden, ME; 2010.

29. Charmel P, Frampton SB: Building the business case for patient-centered care. HFM Magazine 2008, 62(3):80-85.

30. Carolinas Medical Center-Mercy "Engagement and Commitment Put the Hospital on the Fast Track to Planetree Designation. http://planetree.org/ in-the-news/]. Accessed 3.26.14.

31. New York-Presbyterian: The First Planetree Designated ${ }^{\circledR}$ Behavioral Health Organization. [http://planetree.org/in-the-news/]. Accessed 3.26.14.

32. Frampton S, Guastello S: Patient-Centered Care: More than the Sum of Its Parts. Amer J Nurs 2010, 9:49-53.

33. Guastello S: Raising The Bar On Quality: Planetree's designation program takes person-centered care to a new level with evidenced-based criteria and tested measures. Provider 2011, 37(5):35-38.

34. Planetree I: Patient-Centered Hospital Designation Criteria. CT: Derby; 2014.

\section{doi:10.1186/s40639-014-0002-z}

Cite this article as: Guastello and Frampton: Patient-centered care retreats as a method for enhancing and sustaining compassion in action in healthcare settings. Journal of Compassionate Health Care $20141: 2$

\section{Submit your next manuscript to BioMed Central and take full advantage of:}

- Convenient online submission

- Thorough peer review

- No space constraints or color figure charges

- Immediate publication on acceptance

- Inclusion in PubMed, CAS, Scopus and Google Scholar

- Research which is freely available for redistribution 